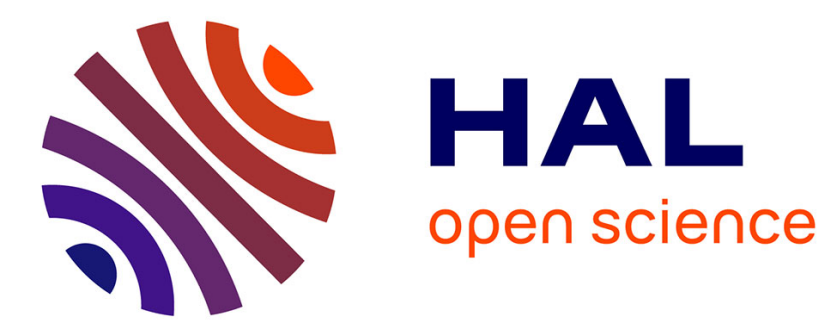

\title{
Measurement of the Michel Parameters and the Average $\tau$-Neutrino Helicity from $\tau$ Decays at LEP
}

\author{
M. Acciarri, O. Adriani, M. Aguilar-Benitez, S. Ahlen, J. Alcaraz, G.
} Alemanni, J. Allaby, A. Aloisio, M G. Alviggi, G. Ambrosi, et al.

\section{- To cite this version:}

M. Acciarri, O. Adriani, M. Aguilar-Benitez, S. Ahlen, J. Alcaraz, et al.. Measurement of the Michel Parameters and the Average $\tau$-Neutrino Helicity from $\tau$ Decays at LEP. Physics Letters B, 1998, 438, pp.405-416. 10.1016/S0920-5632(99)00452-1 . in2p3-00000025

HAL Id: in2p3-00000025

https://hal.in2p3.fr/in2p3-00000025

Submitted on 5 Nov 1998

HAL is a multi-disciplinary open access archive for the deposit and dissemination of scientific research documents, whether they are published or not. The documents may come from teaching and research institutions in France or abroad, or from public or private research centers.
L'archive ouverte pluridisciplinaire HAL, est destinée au dépôt et à la diffusion de documents scientifiques de niveau recherche, publiés ou non, émanant des établissements d'enseignement et de recherche français ou étrangers, des laboratoires publics ou privés. 
EUROPEAN ORGANIZATION FOR NUCLEAR RESEARCH

CERN-EP/98-123

31 July 1998

\title{
Measurement of the Michel Parameters and the Average Tau-Neutrino Helicity from Tau Decays at LEP
}

The L3 Collaboration

\begin{abstract}
Four of the Michel parameters and the average tau-neutrino helicity have been measured by analysing tau decay spectra in $147 \mathrm{pb}^{-1}$ of data collected by the L3 detector. The decays $\tau^{-} \rightarrow \mathrm{e}^{-} \nu_{\tau} \bar{\nu}_{e}, \tau^{-} \rightarrow \mu^{-} \nu_{\tau} \bar{\nu}_{\mu}, \tau^{-} \rightarrow \pi^{-} \nu_{\tau}, \tau^{-} \rightarrow \rho^{-} \nu_{\tau}$ and their charge conjugates were considered. The results: $\rho=0.762 \pm 0.035, \eta=$ $0.27 \pm 0.14, \xi=0.70 \pm 0.16, \xi \delta=0.70 \pm 0.11$ and $\xi_{h}=-1.032 \pm 0.031$ are consistent with a $\mathrm{V}-\mathrm{A}$ structure for the weak charged current and lepton universality.
\end{abstract}

Submitted to Phys. Lett. B 


\section{Introduction}

The Standard Model [1] has proved to be highly successful at explaining the wealth of precise measurements made by the LEP experiments and SLD on the weak neutral current [2]. Similarly, the Standard Model prediction of a V-A Lorentz structure for the weak charged current has been shown to be in excellent agreement with results coming from the precise investigation of muon decay [3]. Nevertheless, a global analysis of the data does leave some room for a non-standard contribution to the charged current [4]. The abundant production and decay of tau pairs at LEP gives an opportunity to search for such deviations from the $\mathrm{V}-\mathrm{A}$ current at higher mass scales than muon decay, as well as to test the Standard Model assumption of lepton universality. The hadronic decay modes of the tau also allow the measurement of the tau-neutrino helicity, imposing a further constraint on the Standard Model. In contrast with experiments at lower energies [5,6], the near perfect anti-correlation of the $\tau^{+} \tau^{-}$helicities and the non-zero tau polarization near the $\mathrm{Z}$ peak facilitate these measurements [7-10].

In this paper, we investigate the Lorentz structure of the weak charged current using $\mathrm{e}^{+} \mathrm{e}^{-} \rightarrow$ $\tau^{+} \tau^{-}$events around the Z peak collected by the L3 detector at LEP. The chosen observables are four of the Michel parameters [11] $\rho, \eta, \xi$ and $\xi \delta$, the average tau-neutrino helicity, expressed as the chirality parameter $\xi_{h}$, and the tau polarization $\mathcal{P}_{\tau}$. The measurement is made using a combined fit to the energy spectra in $\tau^{-} \rightarrow \mathrm{e}^{-} \nu_{\tau} \bar{\nu}_{e}, \tau^{-} \rightarrow \mu^{-} \nu_{\tau} \bar{\nu}_{\mu}, \tau^{-} \rightarrow \pi^{-} \nu_{\tau}$ and $\tau^{-} \rightarrow \rho^{-} \nu_{\tau}$ decays ${ }^{1}$ identified in the 1994-95 data sample. These results are then combined with those of the previous L3 publication [8] to form averages for the full 1991-95 data set.

\section{Measurement method}

Under the most general four-fermion contact interaction Hamiltonian, the charged lepton decay spectra in $\tau^{-} \rightarrow l^{-} \nu_{\tau} \bar{\nu}_{l}$ may be written as [12]:

$$
\begin{aligned}
\frac{1}{\Gamma} \frac{\mathrm{d} \Gamma}{\mathrm{d} x_{l}} & =H_{0}^{l}\left(x_{l}\right)-\mathcal{P}_{\tau} H_{1}^{l}\left(x_{l}\right) \\
& =h_{0}^{l}\left(x_{l}\right)+\eta h_{\eta}^{l}\left(x_{l}\right)+\rho h_{\rho}^{l}\left(x_{l}\right)-\mathcal{P}_{\tau}\left[\xi h_{\xi}^{l}\left(x_{l}\right)+\xi \delta h_{\xi \delta}^{l}\left(x_{l}\right)\right]
\end{aligned}
$$

where $x_{l}=E_{l} / E_{\text {beam }}(l=\mathrm{e}, \mu)$ is the normalized lepton energy and $h^{l}\left(x_{l}\right)$ are kinematical functions.

Similarly, hadronic spectra in $\tau^{-} \rightarrow h^{-} \nu_{\tau}$ are described by

$$
\begin{aligned}
\frac{1}{\Gamma} \frac{\mathrm{d} \Gamma}{\mathrm{d} x_{h}} & =H_{0}^{h}\left(x_{h}\right)-\mathcal{P}_{\tau} H_{1}^{h}\left(x_{h}\right) \\
& =h_{0}^{h}\left(x_{h}\right)-\mathcal{P}_{\tau} \xi_{h} h_{1}^{h}\left(x_{h}\right) .
\end{aligned}
$$

For $\tau^{-} \rightarrow \pi^{-} \nu_{\tau}, x_{\pi}=E_{\pi} / E_{\text {beam }}$. In the case of $\tau^{-} \rightarrow \rho^{-} \nu_{\tau}, x_{\rho}=\omega_{\rho}$ where $\omega_{\rho}$ is a function of the energies and opening angles of the decay products in $\rho^{-} \rightarrow \pi^{-} \pi^{0}$ which optimizes sensitivity to the tau polarization [13]. Qualitatively, negative values of $\omega_{\rho}$ are enriched by left-handed $\tau^{-}$, positive values by right-handed $\tau^{-}$.

\footnotetext{
${ }^{1)}$ Formulae are given for $\tau^{-}$. Charge conjugate decays are implied throughout this paper. Charged pions and kaons are not distinguished.
} 
The joint decay distribution for $\mathrm{e}^{+} \mathrm{e}^{-} \rightarrow \tau^{+} \tau^{-} \rightarrow A^{ \pm} B^{\mp} n \nu(n=2,3,4)$ where A, B are e, $\mu, \pi$ or $\rho$ is

$$
\begin{aligned}
\frac{1}{\Gamma} \frac{d^{2} \Gamma}{d x_{A} d x_{B}} & =H_{0}^{A}\left(x_{A}\right) H_{0}^{B}\left(x_{B}\right)+H_{1}^{A}\left(x_{A}\right) H_{1}^{B}\left(x_{B}\right) \\
& -\mathcal{P}_{\tau}\left[H_{1}^{A}\left(x_{A}\right) H_{0}^{B}\left(x_{B}\right)+H_{0}^{A}\left(x_{A}\right) H_{1}^{B}\left(x_{B}\right)\right]
\end{aligned}
$$

By fitting this form to the correlated tau decay spectra from $\mathrm{e}^{+} \mathrm{e}^{-} \rightarrow \tau^{+} \tau^{-}$, we extract the Michel parameters, the chirality parameter and the average tau polarization up to a sign ambiguity which is solved by reference to other experiments [10,14]. The analysis method follows closely that used in the previous L3 publication [8].

\section{Data sample and event selection}

The data analysed in this paper were recorded by the L3 detector [15] at center-of-mass energies around the $\mathrm{Z}$ peak in 1994-95, and correspond to an integrated luminosity of $78 \mathrm{pb}^{-1}$. A sample of $\mathrm{Z}$ decays to charged lepton pairs is obtained by applying a pre-selection based on event topology and particle multiplicity. Selected events are then divided into two hemispheres by a plane perpendicular to the thrust axis and particle identification is applied to each hemisphere separately.

For efficiency and background estimates, the following Monte Carlo programs are used: KORALZ [16] for $\mathrm{e}^{+} \mathrm{e}^{-} \rightarrow \tau^{+} \tau^{-}(\gamma)$ and $\mathrm{e}^{+} \mathrm{e}^{-} \rightarrow \mu^{+} \mu^{-}(\gamma)$, BHAGENE [17] for $\mathrm{e}^{+} \mathrm{e}^{-} \rightarrow \mathrm{e}^{+} \mathrm{e}^{-}(\gamma)$ and DIAG36 [18] for $\mathrm{e}^{+} \mathrm{e}^{-} \rightarrow \mathrm{e}^{+} \mathrm{e}^{-} \mathrm{ff}$ where ff are $\mathrm{e}^{+} \mathrm{e}^{-}, \mu^{+} \mu^{-}, \tau^{+} \tau^{-}$or $\mathrm{q} \overline{\mathrm{q}}$. Generated events are passed through a full detector simulation based on the GEANT [19] program. The number of Monte Carlo events for each process is about eight times larger than the data sample.

\section{Particle identification}

Particle identification is restricted to the barrel region of the detector $(|\cos \theta|<0.7)$. Electrons are identified as a track reconstructed in the central tracker that is matched to a narrow shower in the electromagnetic calorimeter. There must be little or no energy in the hadron calorimeter and no associated track in the muon spectrometer. Muons have a track in the muon spectrometer, reconstructed from three precision chamber segments, that extrapolates back to the interaction point with a minimum ionizing response in the calorimeters. There must be an associated track in the inner tracker.

Hadrons are identified using the central tracker and the calorimeters. Tracking information is used to identify the impact point of a charged particle at the electromagnetic calorimeter. An algorithm [20] is applied to disentangle overlapping neutral calorimetric clusters in the vicinity of the impact point and a hadronic shower, whose shape is nearly energy independent, is subtracted from the energy deposit. Electromagnetic neutral identification criteria are applied to the remaining local maxima.

Shower shape and energy cuts are applied to neutral clusters to identify neutral pions via their decay into two photons. For pion energies below $10 \mathrm{GeV}$, a large fraction of the photons are spatially resolved. Above this energy the showers from the two photons overlap to form a single neutral electromagnetic cluster.

The decays $\tau^{-} \rightarrow \pi^{-} \nu_{\tau}$ are identified as hemispheres that are inconsistent with an electron or muon, containing one charged track, no $\pi^{0}$ candidates and calorimetric energy well matched 
with the track momentum. The decays $\tau^{-} \rightarrow \rho^{-} \nu_{\tau}$ are identified as hemispheres with one charged track and one $\pi^{0}$ candidate, a total calorimetric energy greater than $5 \mathrm{GeV}$ and an invariant mass of the charged-neutral pair within the range $0.45-1.20 \mathrm{GeV}$.

\section{Event selection}

Events with at least one identified tau decay are retained and subjected to loose cuts that correlate the information in both hemispheres in order to reduce contamination from non-tau events while introducing minimal distortion of the measured decay spectra. Bhabha, dimuon and two-photon backgrounds are reduced to the level of a few percent by applying cuts on the total visible energy and jet acollinearity and aplanarity. Cosmic muons are rejected by cuts on scintillator timing and the distance of closest approach (DCA) of tracks to the interaction point.

Surviving events are classified according to the tau decay candidate in each hemisphere: ee, $\mathrm{e} \mu, \mathrm{e} \pi, \mathrm{e} \rho, \mathrm{e} X, \mu \mu, \mu \pi, \mu \rho, \mu X, \pi \pi, \pi \rho, \pi X, \rho \rho$ and $\rho X$ where $X$ represents a hemisphere with no identified tau decay. Backgrounds in each channel coming from misidentified tau decays are estimated from simulated $\mathrm{Z} \rightarrow \tau^{+} \tau^{-}$events. Bhabha, dimuon and two-photon background shapes are taken from Monte Carlo and normalized using the number of such events estimated to be present in the selected data sample. Background from cosmic muons is estimated using a control sample of data selected by loosening the cut on the track DCA.

The measured spectra are fitted over the full acceptance range except in the case of electrons and muons, where the fit is restricted to regions where the background is small. The fit range for each channel, number of selected events, selection efficiency and the tau and non-tau background fractions are shown in Table 1.

\section{Fit procedure}

We perform a simultaneous measurement of $\rho, \eta, \xi, \xi \delta, \xi_{h}$ and $\mathcal{P}_{\tau}$ using a binned maximumlikelihood fit to the decay spectra of selected events. In the case where only one hemisphere has an identified tau decay, the one-dimensional spectrum of the identified particle is used. If both hemispheres are identified the decay variables of both particles are used to form a two-dimensional correlated decay spectrum.

The fit procedure is described in detail in [8]. The likelihood function is

$$
\mathcal{L}=\prod_{i, j} \frac{\left(w_{i j}(\alpha)+b_{i j}\right)^{n_{i j}} e^{-\left(w_{i j}(\alpha)+b_{i j}\right)}}{n_{i j} !},
$$

where $n_{i j}$ is the number of data events observed in the $j$-th bin of the $i$-th decay mode, $w_{i j}(\alpha)$ is the expected number of signal events for a particular set of parameters $\alpha=\left\{\rho, \eta, \xi, \xi \delta, \xi_{h}, \mathcal{P}_{\tau}\right\}$ and $b_{i j}$ is the sum of tau and non-tau background. The dependence of the tau-background on $\alpha$ is taken into account.

Each $w_{i j}(\alpha)$ is given by a convolution of the appropriate kinematical functions with the detector resolution and acceptance. The total number of expected signal and background events is fixed to the total number of events selected in the data for each decay channel.

The kinematical functions are obtained using linear combinations of decay spectra generated by KORALZ with a modified version of the TAUOLA [21] $\tau$ decay library that allows the generation of samples with any combination of Michel parameters. Functions for $\mu, \pi$ and $\rho$ 


\begin{tabular}{|c|c|c|c|c|c|}
\hline \multirow[t]{2}{*}{ Channel } & \multirow[t]{2}{*}{ Fit Range } & \multirow[t]{2}{*}{ Events } & \multirow{2}{*}{$\begin{array}{l}\epsilon(\%) \\
\text { in } 4 \pi\end{array}$} & \multicolumn{2}{|c|}{$\operatorname{Bkg}(\%)$} \\
\hline & & & & $\tau$ & non- $\tau$ \\
\hline ee & $x_{e}[0.25,0.75]$ & 558 & 19.4 & 4.2 & 7.0 \\
\hline $\mathrm{e} \mu$ & $\begin{array}{l}x_{e}[0.05,0.95] \\
x_{\mu}[0.05,0.95]\end{array}$ & 1574 & 33.7 & 3.1 & 0.1 \\
\hline $\mathrm{e} \pi$ & $\begin{array}{l}x_{e}[0.05,0.95] \\
x_{\pi}[0,1.4]\end{array}$ & 1489 & 36.3 & 12.3 & 2.3 \\
\hline $\mathrm{e} \rho$ & $\begin{array}{l}x_{e}[0.05,0.95] \\
\omega_{\rho}[-1,1]\end{array}$ & 2817 & 34.1 & 14.9 & 0.4 \\
\hline $\mathrm{e} X$ & $x_{e}[0.05,0.95]$ & 6176 & 66.5 & 2.5 & 4.3 \\
\hline$\mu \mu$ & $x_{\mu}[0.05,0.75]$ & 437 & 22.0 & 0.6 & 1.0 \\
\hline$\mu \pi$ & $\begin{array}{l}x_{\mu}[0.05,0.95] \\
x_{\pi}[0,1.4]\end{array}$ & 1002 & 27.6 & 11.5 & 0.6 \\
\hline$\mu \rho$ & $\begin{array}{l}x_{\mu}[0.05,0.95] \\
\omega_{\rho}[-1,1]\end{array}$ & 1892 & 26.9 & 13.2 & 0.3 \\
\hline$\mu X$ & $x_{\mu}[0.05,0.95]$ & 3897 & 49.1 & 0.7 & 1.0 \\
\hline$\pi \pi$ & $x_{\pi}[0,1.4]$ & 456 & 30.8 & 20.6 & 2.1 \\
\hline$\pi \rho$ & $\begin{array}{l}x_{\pi}[0,1.4] \\
\omega_{\rho}[-1,1]\end{array}$ & 1921 & 30.2 & 22.3 & 0.8 \\
\hline$\pi X$ & $x_{\pi}[0,1.4]$ & 4088 & 58.4 & 12.0 & 1.1 \\
\hline$\rho \rho$ & $\omega_{\rho}[-1,1]$ & 1816 & 28.8 & 23.4 & 0.1 \\
\hline$\rho X$ & $\omega_{\rho}[-1,1]$ & 7500 & 55.5 & 12.8 & 0.3 \\
\hline Total & & 35623 & & & \\
\hline
\end{tabular}

Table 1: Fit ranges for each channel, numbers of events selected, selection efficiencies $\epsilon$ and $\tau$ and non- $\tau$ background fractions. Only events within the fit range are included in this table. 


\begin{tabular}{|r|rrrrr|}
\hline & $\eta$ & $\xi$ & $\xi \delta$ & $\xi_{h}$ & $\mathcal{P}_{\tau}$ \\
\hline$\rho$ & 0.54 & -0.20 & -0.27 & -0.14 & 0.22 \\
$\eta$ & & 0.13 & 0.04 & 0.01 & 0.03 \\
$\xi$ & & & 0.12 & 0.11 & 0.06 \\
$\xi \delta$ & & & & 0.16 & -0.15 \\
$\xi_{h}$ & & & & & -0.30 \\
\hline
\end{tabular}

Table 2: Correlation coefficients for the fit to 1994-95 data.

are shown in Figure 1. Figure 1 also shows the effect of the convolution of the kinematical functions for $\tau^{-} \rightarrow \pi^{-} \nu_{\tau}$ and $\tau^{-} \rightarrow \rho^{-} \nu_{\tau}$ with the detector resolution and acceptance.

\section{Results}

The results of the fit to the leptonic and hadronic decay channels in the 1994-95 data are:

$$
\begin{array}{rllll}
\rho & =0.724 & \pm 0.043 & \pm 0.021 & (0.75) \\
\eta & =0.26 & \pm 0.19 & \pm 0.08 & (0) \\
\xi & =0.51 & \pm 0.19 & \pm 0.09 & (1) \\
\xi \delta & =0.62 & \pm 0.14 & \pm 0.06 & (0.75) \\
\xi_{h} & =-1.065 & \pm 0.033 & \pm 0.016 & (-1) \\
\mathcal{P}_{\tau} & =-0.173 & \pm 0.016 & \pm 0.014 &
\end{array}
$$

where the first error is statistical and the second systematic. For comparison, the Standard Model predictions are shown in parentheses. The correlation coefficients between the parameters are shown in in Table 2. Estimation of the systematic errors is described below. Examples of the fit result in comparison to the data are shown in Figures 2 and 3.

In a separate analysis that considers only the hadronic decay channels but includes a reanalysis of the 1991-93 data, the chirality parameter $\xi_{h}$ and the tau polarization $\mathcal{P}_{\tau}$ have been independently determined [22]. Good agreement between the two analyses is seen.

\section{Systematic errors}

We considered the following sources of systematic error: event selection, background estimation, the energy calibration of the detector, and the use of finite Monte Carlo statistics. These sources are considered to be independent.

To determine the systematic error due to event selection, we varied the correlated cuts between hemispheres by amounts corresponding to the estimated inaccuracy of the Monte Carlo simulation for each cut variable. The error due to the uncertainty in the background coming from mis-identified tau-decays was estimated by varying the largest decay branching fractions of the tau by their experimental errors [4]. Non-tau background systematics were estimated by varying the normalization of all non-tau backgrounds by their statistical errors.

The error due to detector calibration uncertainties was estimated by varying the appropriate energy scales by their known uncertainty. The momentum scale of the central tracker is known to $0.5 \%$ from a comparison of its muon momentum measurements and those of the muon spectrometer. The accuracy of the energy scale of the electromagnetic calorimeter is known 


\begin{tabular}{|r|r|r|r|r|r|r|}
\hline Uncertainty & $\Delta \rho$ & $\Delta \eta$ & $\Delta \xi$ & $\Delta \xi \delta$ & $\Delta \xi_{h}$ & $\Delta \mathcal{P}_{\tau}$ \\
\hline Selection & 0.003 & 0.01 & 0.04 & 0.01 & 0.005 & 0.003 \\
Background & 0.010 & 0.03 & 0.02 & 0.02 & 0.006 & 0.003 \\
Calibration & 0.010 & 0.02 & 0.04 & 0.03 & 0.007 & 0.012 \\
MC statistics & 0.015 & 0.07 & 0.07 & 0.05 & 0.012 & 0.006 \\
\hline Total & 0.021 & 0.08 & 0.09 & 0.06 & 0.016 & 0.014 \\
\hline
\end{tabular}

Table 3: Summary of systematic errors for 1994-95 data.

\begin{tabular}{|r|rrrrr|}
\hline & $\eta$ & $\xi$ & $\xi \delta$ & $\xi_{h}$ & $\mathcal{P}_{\tau}$ \\
\hline$\rho$ & 0.50 & -0.19 & -0.26 & -0.21 & 0.32 \\
$\eta$ & & 0.13 & 0.06 & 0.00 & 0.03 \\
$\xi$ & & & 0.07 & 0.11 & 0.11 \\
$\xi \delta$ & & & & 0.25 & -0.19 \\
$\xi_{h}$ & & & & & -0.35 \\
\hline
\end{tabular}

Table 4: Correlation coefficients for the combined 1991-95 results.

from the position of the $\pi^{0}$ peak to be $0.5 \%$ below $5 \mathrm{GeV}$, and from Bhabha events to be $0.05 \%$ at $45 \mathrm{GeV}$. The energy scale of the hadron calorimeter is accurate to $1 \%$, as estimated from the peak position of the $\rho$ resonance. The momentum scale of the muon spectrometer is known from dimuon events to be accurate to $0.05 \%$. Below $5 \mathrm{GeV}$ the muon momentum measurement is dominated by energy loss in the calorimeters and the scale is known to $0.5 \%$. At low energy, a cross-check of the calibration of the central tracker with the electromagnetic calorimeter and the muon spectrometer is performed using electrons and muons from tau decays and two-photon events.

The contribution of each systematic error is given in Table 3.

\section{Combination of results}

The results obtained from the fit to the 1994-95 data are combined with those of the previous L3 publication [8] to obtain averages for the 1991-95 data set, corresponding to a total integrated luminosity of $147 \mathrm{pb}^{-1}$ :

$$
\begin{aligned}
\rho & =0.762 & & \pm 0.035 \\
\eta & =0.27 & & \pm 0.14 \\
\xi & =0.70 & & \pm 0.16 \\
\xi \delta & =0.70 & & \pm 0.11 \\
\xi_{h} & =-1.032 & & \pm 0.031 \\
\mathcal{P}_{\tau} & =-0.164 & & \pm 0.016
\end{aligned}
$$

The combined correlation coefficients are given in Table 4. In making this average, the systematics due to event selection were considered to be completely correlated between the two sets of measurements. The remaining systematic errors were treated as independent.

The values obtained for the Michel parameters are in agreement with a V-A structure of the weak charged current in decays of the tau lepton and support the Standard Model assumption of 
lepton universality. The measured chirality parameter $\xi_{h}$ is in agreement with the assumption of only left-handed neutrinos in semileptonic tau decays. The mean tau polarization is consistent with the results from a separate analysis of the L3 data [23] that focuses on the measurement of neutral couplings and assumes a V-A structure for the tau decay vertex.

\section{Acknowledgements}

We wish to express our gratitude to the CERN accelerator divisions for the excellent performance of the LEP machine. We acknowledge the contributions of all the engineers and technicians who have participated in the construction and maintenance of this experiment. 


\section{References}

[1] S. Glashow, Nucl. Phys. 22 (1961) 579;

A. Salam and J. Ward, Phys. Lett. 13 (1964) 168;

S. Weinberg, Phys. Rev. Lett. 19 (1967) 1264;

S.L. Glashow, I. Illiopoulos, L. Maiani, Phys. Rev. D 2 (1970) 1285.

[2] ALEPH, DELPHI, L3 and OPAL Collaborations, LEP Electroweak Working Group and SLD Heavy Flavour Group, D. Abbaneo et al., A Combination of Preliminary Electroweak Measurements and Constraints on the Standard Model, CERN-PPE/97-154 (1997).

[3] H. Burkhard et al., Phys. Lett. B 160 (1985) 343;

B. Balke et al., Phys. Rev. D 37 (1988) 587;

W. Fetscher, H. Gerber and K. Johnson, Phys. Lett. B 173 (1986) 102.

[4] Particle Data Group, C. Caso et al., Eur. Phys. Jour. C 3 (1998) 1.

The V-A structure of muon and tau decays is discussed in pp. 282-286 and pp. 303-306.

[5] ARGUS Collaboration, H. Albrecht et al., DESY 97-194 (1997), Submitted to Phys. Lett. B.

[6] CLEO Collaboration, J. Alexander et al., Phys. Rev. D 56 (1997) 5320.

[7] ALEPH Collaboration, D. Buskulic et al., Phys. Lett. B 346 (1995) 379.

[8] L3 Collaboration, M. Acciarri et al., Phys. Lett. B 377 (1996) 313.

[9] OPAL Collaboration, K. Ackerstaff et al., CERN-EP/98-104 (1998), Submitted to Eur. Phys. Jour. C.

[10] SLD Collaboration, K. Abe et al., Phys. Rev. Lett. 78 (1997) 4641.

[11] L. Michel, Proc. Phys. Soc. A 63 (1950) 514;

C. Bouchiat and L. Michel, Phys. Rev. 106 (1957) 170;

T. Kinoshita and A. Sirlin, Phys. Rev. 107 (1957) 593;

T. Kinoshita and A. Sirlin, Phys. Rev. 108 (1957) 844.

[12] C. Nelson, Phys. Rev. D 40 (1989) 123, erratum Phys. Rev D 41 (1990) 2327;

W. Fetscher, Phys. Rev. D 42 (1990) 1544;

R. Alemany et al., Nucl. Phys. B 379 (1992) 3.

[13] M. Davier et al., Phys. Lett. B 306 (1993) 411.

[14] ARGUS Collaboration, H. Albrecht et al., Phys. Lett. B 250 (1990) 164.

[15] L3 Collaboration, B. Adeva et al., Nucl. Inst. and Meth. A 289 (1990) 35;

M. Chemarin et al., Nucl. Inst. and Meth. A 349 (1994) 345;

M. Acciarri et al., Nucl. Inst. and Meth. A 351 (1994) 300;

G. Basti et al., Nucl. Inst. and Meth. A 374 (1996) 293;

I. C. Brock et al., Nucl. Inst. and Meth. A 381 (1996) 236;

A. Adam et al., Nucl. Inst. and Meth. A 383 (1996) 342.

[16] S. Jadach et al., Comp. Phys. Comm. 66 (1991) 276. 
[17] J. H. Field, Phys. Lett. B 323 (1994) 432;

J. H. Field and T. Riemann, Comp. Phys. Comm. 94 (1996) 53.

[18] F. A. Berends, P. H. Daverfeldt and R. Kleiss, Nucl. Phys. B 253 (1985) 441.

[19] The L3 detector simulation is based on GEANT Version 3.15, R. Brun et al., CERN DD/EE/84-1 (Revised), September 1987.

Hadronic interactions are simulated by the GHEISHA program, H. Fesefeldt, RWTH Aachen Report PITHA 85/02 (1985).

[20] L3 Collaboration, M. Acciarri et al., Phys. Lett. B 341 (1994) 245.

[21] M. Schmidtler, University Karlsruhe IEKP-KA/93-14 (1993).

[22] R. Moore, Measurement of the Chirality Parameter in the Charged-Current Coupling of the Tau Lepton, Ph.D. thesis, University of Michigan, May 1998 (unpublished).

[23] L3 Collaboration, M. Acciarri et al., Phys. Lett. B 429 (1998) 387. 


\section{The L3 Collaboration:}

M.Acciarri ${ }^{27}$ O.Adriani ${ }^{16}$ M.Aguilar-Benitez ${ }^{26}$ S.Ahlen $^{11}{ }^{1}$ J.Alcaraz ${ }^{26}$ G.Alemanni ${ }^{22}$ J.Allaby, ${ }^{17}$ A.Aloisio ${ }^{29}$ M.G.Alviggi ${ }^{29}$ G.Ambrosi, ${ }^{19}$ H.Anderhub, ${ }^{48}$ V.P.Andreev ${ }^{37}$ T.Angelescu, ${ }^{13}$ F.Anselmo, ${ }^{9}$ A.Arefiev ${ }^{28}$ T.Azemoon, ${ }^{3}$ T.Aziz, ${ }^{10}$ P.Bagnaia, ${ }^{36}$ L.Baksay, ${ }^{43}$ R.C.Ball, ${ }^{3}$ S.Banerjee, ${ }^{10}$ Sw.Banerjee, ${ }^{10}$ K.Banicz, ${ }^{45}$ A.Barczyk, ${ }^{48,46}$ R.Barillère ${ }^{17}$ L.Barone ${ }^{36}$ P.Bartalini ${ }^{22}$ A.Baschirotto, ${ }^{27}$ M.Basile, ${ }^{9}$ R.Battiston ${ }^{33}$ A.Bay ${ }^{22}$ F.Becattini, ${ }^{16}$ U.Becker, ${ }^{15}$ F.Behner, ${ }^{48}$ J.Berdugo, ${ }^{26}$ P.Berges ${ }^{15}$ B.Bertucci ${ }^{33}$ B.L.Betev, ${ }^{48}$ S.Bhattacharya ${ }^{10}$ M.Biasini ${ }^{33}$ A.Biland, ${ }^{48}$ G.M.Bilei ${ }^{33}$ J.J.Blaising, ${ }^{4}$ S.C.Blyth ${ }^{34}$ G.J.Bobbink ${ }^{2}$ R.Bock, ${ }^{1}$ A.Böhm, ${ }^{1}$ L.Boldizsar, ${ }^{14}$ B.Borgia,${ }^{17,36}$ D.Bourilkov, ${ }^{48}$ M.Bourquin, ${ }^{19}$ D.Boutigny, ${ }^{4}$ S.Braccini, ${ }^{19}$ J.G.Branson ${ }^{39}$ V.Brigljevic, ${ }^{48}$ I.C.Brock, ${ }^{34}$ A.Buffini ${ }^{16}$ A.Buijs, ${ }^{44}$ J.D.Burger, ${ }^{15}$ W.J.Burger ${ }^{33}$ J.Busenitz, ${ }^{43}$ X.D.Cai ${ }^{15}$ M.Campanelli, ${ }^{48}$ M.Capell $^{15}{ }^{\text {G.Cara Romeo, }}{ }^{9}$ G.Carlino, ${ }^{29}$ A.M.Cartacci ${ }^{16}$ J.Casaus, ${ }^{26}$ G.Castellini ${ }^{16}$ F.Cavallari ${ }^{36}$ N.Cavallo ${ }^{29}$ C.Cecchi ${ }^{19}$ M.Cerrada ${ }^{26}$ F.Cesaroni ${ }^{23}$ M.Chamizo, ${ }^{26}$ Y.H.Chang, ${ }^{50}$ U.K.Chaturvedi, ${ }^{18}$ M.Chemarin ${ }^{25}$ A.Chen ${ }^{50}$ G.Chen, ${ }^{7}$ G.M.Chen, H.F.Chen ${ }^{20}$ H.S.Chen, ${ }^{7}$ M.Chen, ${ }^{15}$ G.Chiefari ${ }^{29}$ C.Y.Chien, ${ }^{5}$ L.Cifarelli ${ }^{38}$ F.Cindolo, C.Civinini ${ }^{16}{ }^{9}$ I.Clare, ${ }^{15}$ R.Clare, ${ }^{15}$ G.Coignet, ${ }^{4}$ A.P.Colijn, 2 N.Colino, ${ }^{26}$ S.Costantini, ${ }^{8}$ F.Cotorobai ${ }^{13}$ B.de la Cruz ${ }^{26}$ A.Csilling ${ }^{14}$ T.S.Dai ${ }^{15}$ R.D'Alessandro, ${ }^{16}$ R.de Asmundis, ${ }^{29}$ A.Degré, ${ }^{4}$ K.Deiters, ${ }^{46}$ P.Denes, ${ }^{35}$ F.DeNotaristefani, ${ }^{36}$ M.Diemoz, ${ }^{36}$ D.van Dierendonck, F.Di Lodovico, ${ }^{48}$ C.Dionisi, ${ }^{1,36}$ M.Dittmar ${ }^{48}$ A.Dominguez, ${ }^{39}$ A.Doria, ${ }^{29}$ M.T.Dova, ${ }^{18, \sharp}$ E.Drago, ${ }^{29}$ D.Duchesneau, ${ }^{4}$ P.Duinker, ${ }^{2}$ I.Duran,${ }^{40}$ S.Easo ${ }^{33}$ H.El Mamouni ${ }^{25}$ A.Engler ${ }^{34}$ F.J.Eppling, ${ }^{15}$ F.C.Erné ${ }^{2}$ P.Extermann ${ }^{19}$ M.Fabre ${ }^{46}$ R.Faccini ${ }^{36}$ M.A.Falagan ${ }^{26}$ S.Falciano ${ }^{36}$ A.Favara ${ }^{16}{ }^{16}$ J.Fay, ${ }^{25}$ O.Fedin ${ }^{37}$ M.Felcini ${ }^{48}$ T.Ferguson, ${ }^{34}$ F.Ferroni ${ }^{36}$ H.Fesefeldt, ${ }^{1}$ E.Fiandrini, ${ }^{33}$ J.H.Field ${ }^{19}$ F.Filthaut ${ }^{17}$ P.H.Fisher ${ }^{15}$ I.Fisk ${ }^{39}$ G.Forconi ${ }^{15}$ L.Fredj ${ }^{19}$ K.Freudenreich, ${ }^{48}$ C.Furetta ${ }^{27}$ Yu.Galaktionov, ${ }^{28,15}$ S.N.Ganguli, ${ }^{10}$ P.Garcia-Abia, ${ }^{6}$ M.Gataullin ${ }^{32}$ S.S.Gau, ${ }^{12}$ S.Gentile, ${ }^{36}$ J.Gerald ${ }^{5}$ N.Gheordanescu, ${ }^{13}$ S.Giagu, ${ }^{36}$ S.Goldfarb, ${ }^{22}$ J.Goldstein, ${ }^{11}$ Z.F.Gong, ${ }^{20}$ A.Gougas, G.Gratta, ${ }^{32}$ M.W.Gruenewald, ${ }^{8}$ R.van Gulik, V.K.Gupta ${ }^{35}$ A.Gurtu ${ }^{10}$ L.J.Gutay, ${ }^{45}$ D.Haas, ${ }^{6}$ B.Hartmann, A.Hasan ${ }^{30}$ D.Hatzifotiadou, ${ }^{9}$ T.Hebbeker, ${ }^{8}$ A.Hervé ${ }^{17}$ P.Hidas, ${ }^{14}$ J.Hirschfelder, ${ }^{34}$ W.C.van Hoek ${ }^{31}$ H.Hofer ${ }^{48}$ H.Hoorani ${ }^{34}$ S.R.Hou ${ }^{50}$ G.Hu, ${ }^{5}$ I.Iashvili, ${ }^{47}$ B.N.Jin, ${ }^{7}$ L.W.Jones, ${ }^{3}$ P.de Jong, ${ }^{17}$ I.Josa-Mutuberria, ${ }^{26}$ A.Kasser, ${ }^{22}$ R.A.Khan ${ }^{18}$ D.Kamrad, ${ }^{47}$ J.S.Kapustinsky, ${ }^{24}$ Y.Karyotakis, ${ }^{4}$ M.Kaur, ${ }^{18, \diamond}$ M.N.Kienzle-Focacci, ${ }^{19}$ D.Kim, ${ }^{36}$ D.H.Kim ${ }^{42}$ J.K.Kim ${ }^{42}$ S.C.Kim ${ }^{42}$ W.W.Kinnison, ${ }^{24}$ A.Kirkby ${ }^{32}$ D.Kirkby, ${ }^{32}$ J.Kirkby, ${ }^{17}$ D.Kiss,${ }^{14}$ W.Kittel ${ }^{31}$ A.Klimentov ${ }^{15,28}$ A.C.König, ${ }^{31}$ A.Kopp ${ }^{47}$ I.Korolko, ${ }^{28}$ V.Koutsenko, ${ }^{15,28}$ R.W.Kraemer, ${ }^{34}$ W.Krenz, ${ }^{1}$ A.Kunin, ${ }^{15,28}$ P.Lacentre ${ }^{47, b, \sharp}$ P.Ladron de Guevara ${ }^{26}$ G.Landi ${ }^{16}$ C.Lapoint ${ }^{15}$ K.Lassila-Perini ${ }^{48}$ P.Laurikainen ${ }^{21}$ A.Lavorato, ${ }^{38}$ M.Lebeau, ${ }^{17}$ A.Lebedev, ${ }^{15}$ P.Lebrun ${ }^{25}$ P.Lecomte, ${ }^{48}$ P.Lecoq ${ }^{17}$ P.Le Coultre ${ }^{48}$ H.J.Lee ${ }^{8}$ C.Leggett, ${ }^{3}$ J.M.Le Goff ${ }^{17}$ R.Leiste ${ }^{47}$ E.Leonardi, ${ }^{36}$ P.Levtchenko, ${ }^{37}$ C.Li ${ }^{20}$ C.H.Lin ${ }^{50}$ W.T.Lin ${ }^{50}$ F.L.Linde,${ }^{2,17}$ L.Lista ${ }^{29}$ Z.A.Liu, W.Lohmann, ${ }^{47}$ E.Longo, ${ }^{36}$ W.Lu, ${ }^{32}$ Y.S.Lu, ${ }^{7}$ K.Lübelsmeyer, ${ }^{1}$ C.Luci ${ }^{17,36}$ D.Luckey, ${ }^{15}$ L.Luminari ${ }^{36}$ W.Lustermann ${ }^{48}$ W.G.Ma ${ }^{20}$ M.Maity, ${ }^{10}$ G.Majumder ${ }^{10}$ L.Malgeri ${ }^{17}$ A.Malinin, ${ }^{28}$ C.Maña ${ }^{26}$ D.Mangeol, ${ }^{31}$ P.Marchesini, ${ }^{48}$ G.Marian, ${ }^{43}$, A.Marin, ${ }^{11}$ J.P.Martin ${ }^{25}$ F.Marzano, ${ }^{36}$ G.G.G.Massaro, K.Mazumdar, ${ }^{10}$ S.Mele, ${ }^{17}$ L.Merola, ${ }^{29}$ M.Meschini, ${ }^{16}$ W.J.Metzger, ${ }^{31}$ M.von der Mey, ${ }^{1}$ Y.Mi, ${ }^{22}$ D.Migani, ${ }^{9}$ A.Mihul, ${ }^{13}$ A.J.W.van Mil ${ }^{31}$ H.Milcent, ${ }^{17}$ G.Mirabellii, ${ }^{36}$ J.Mnich, ${ }^{17}$ P.Molnar, ${ }^{8}$ B.Monteleoni ${ }^{16}$ R.Moore, ${ }^{3}$ T.Moulik, ${ }^{10}$ R.Mount ${ }^{32}$ G.S.Muanza ${ }^{25}$ F.Muheim ${ }^{19}$ A.J.M.Muijs, ${ }^{2}$ S.Nahn, ${ }^{15}$ M.Napolitano, ${ }^{29}$ F.Nessi-Tedaldi, ${ }^{48}$ H.Newman ${ }^{32}$ T.Niessen, A.Nippe ${ }^{22}$ A.Nisati ${ }^{36}$ H.Nowak ${ }^{47}$ Y.D.OH ${ }^{42}$ G.Organtini ${ }^{36}$ R.Ostonen ${ }^{21}$ C.Palomares ${ }^{26}$ D.Pandoulas, ${ }^{1}$ S.Paoletti ${ }^{36,17}$ P.Paolucci ${ }^{29}$ H.K.Park ${ }^{34}$ I.H.Park ${ }^{42}$ G.Pascale, ${ }^{36}$ G.Passaleva,${ }^{17}$ S.Patricelli ${ }^{29}{ }^{2}$ T.Paul ${ }^{12}$ M.Pauluzzi ${ }^{33}$ C.Paus ${ }^{17}$ F.Pauss ${ }^{48}$ D.Peach ${ }^{17}$ M.Pedace ${ }^{36}$ Y.J.Pei ${ }^{1}$ S.Pensotti ${ }^{27}$ D.Perret-Gallix, ${ }^{4}$ B.Petersen ${ }^{31}$ S.Petrak, ${ }^{8}$ A.Pevsner, ${ }^{5}$ D.Piccolo, ${ }^{29}$ M.Pieri, ${ }^{16}$ P.A.Piroué, ${ }^{35}$ E.Pistolesi ${ }^{27}$ V.Plyaskin ${ }^{28}$ M.Pohl $^{48}$ V.Pojidaev ${ }^{28,16}$ H.Postema, ${ }^{15}$ J.Pothier $^{17}$ N.Produit ${ }^{19}$ D.Prokofiev, ${ }^{37}$ J.Quartieri ${ }^{38}$ G.Rahal-Callot ${ }^{48}$ N.Raja, ${ }^{10}$ P.G.Rancoita ${ }^{27}$ M.Rattaggi, ${ }^{27}$ G.Raven ${ }^{39}$ P.Razis, ${ }^{30}$ D.Ren ${ }^{48}$ M.Rescigno, ${ }^{36}$ S.Reucroft, ${ }^{12}$ T.van Rhee, ${ }^{44}$ S.Riemann, ${ }^{47}$ K.Riles, ${ }^{3}$ O.Rind, ${ }^{3}$ A.Robohm, J.Rodin, ${ }^{43}$ B.P.Roe, ${ }^{3}$ L.Romero, ${ }^{26}$ S.Rosier-Lees, ${ }^{4}$ Ph.Rosselet ${ }^{22}$ S.Roth, ${ }^{1}$ J.A.Rubio, ${ }^{17}$ D.Ruschmeier, ${ }^{8}$ H.Rykaczewski, ${ }^{48}$ S.Sakar, ${ }^{36}$ J.Salicio, ${ }^{17}$ E.Sanchez ${ }^{26}$ M.P.Sanders ${ }^{31}$ M.E.Sarakinos ${ }^{21}$ G.Sauvage, C.Schäfer ${ }^{1}$ V.Schegelsky, ${ }^{37}$ S.Schmidt-Kaerst, D.Schmitz, ${ }^{1}$ M.Schneegans, ${ }^{4}$ N.Scholz, ${ }^{48}$ H.Schopper, ${ }^{49}$ D.J.Schotanus, ${ }^{31}$ J.Schwenke, G.Schwering, C.Sciacca, ${ }^{19}$ D.Sciarrino, ${ }^{19}$ L.Servoli, ${ }^{33}$ S.Shevchenko, ${ }^{32}$ N.Shivarov, ${ }^{41}$ V.Shoutko, ${ }^{28}$ J.Shukla ${ }^{24}$ E.Shumilov, ${ }^{28}$ A.Shvorob, ${ }^{32}$ T.Siedenburg, ${ }^{1}$ D.Son, ${ }^{42}$ V.Soulimov ${ }^{29}$ B.Smith, ${ }^{15}$ P.Spillantini ${ }^{16}$ M.Steuer, ${ }^{15}$ D.P.Stickland, ${ }^{35}$ H.Stone, ${ }^{35}$ B.Stoyanov, ${ }^{41}$ A.Straessner, ${ }^{1}$ K.Sudhakar, ${ }^{10}$ G.Sultanov, ${ }^{18}$ L.Z.Sun ${ }^{20}$ G.F.Susinno, ${ }^{19}$ H.Suter, ${ }^{48}$ J.D.Swain ${ }^{18}$ X.W.Tang, ${ }^{7}$ L.Tauscher, ${ }^{6}$ L.Taylor, ${ }^{12}$ C.Timmermans, ${ }^{31}$ Samuel C.C.Ting, ${ }^{15}$ S.M.Ting, ${ }^{15}$ S.C.Tonwar ${ }^{10}$ J.Tóth ${ }^{14}$ C.Tully ${ }^{35}$ K.L.Tung, ${ }^{7}$ Y.Uchida, ${ }^{15}$ J.Ulbrichtt, ${ }^{48}$ E.Valente ${ }^{36}$ G.Vesztergombi ${ }^{14}$ I.Vetlitsky $^{28}$ G.Viertel ${ }^{48}$ S.Villa, ${ }^{12}$ M.Vivargent, ${ }^{4}$ S.Vlachos, ${ }^{6}$ H.Vogel ${ }^{34}$ H.Vogt ${ }^{47}{ }^{4}$ I.Vorobiev, ${ }^{17,28}$ A.A.Vorobyov ${ }^{37}$ A.Vorvolakos ${ }^{30}$ M.Wadhwa, W.Wallraff, J.C.Wang, ${ }^{15}$ X.L.Wang, ${ }^{20}$ Z.M.Wang, ${ }^{20}$ A.Weber, ${ }^{1}$ S.X.Wu ${ }^{15}{ }^{15}$ S.Wynhoff, ${ }^{1}$ J.Xu, ${ }^{11}$ Z.Z.Xu ${ }^{20}$ B.Z.Yang, ${ }^{20}$ C.G.Yang, H.J.Yang, M.Yang, ${ }^{7}$ J.B.Ye, ${ }^{20}{ }^{7}$ S.C.Yeh ${ }^{51}$ J.M.You, ${ }^{34}$ An.Zalite, ${ }^{37}$ Yu.Zalite, ${ }^{37}$ P.Zemp ${ }^{48}$ Y.Zeng, Z.P.Zhang, ${ }^{20}$ B.Zhou, ${ }^{11}$ Y.Zhou, ${ }^{3}$ G.Y.Zhu, R.Y.Zhu, ${ }^{32}$ A.Zichichi, ${ }^{9,17}{ }^{78}$ F.Ziegler, ${ }^{47}$ G.Zilizi. ${ }^{43,}$ 
1 I. Physikalisches Institut, RWTH, D-52056 Aachen, FRG ${ }^{\S}$

III. Physikalisches Institut, RWTH, D-52056 Aachen, FRG ${ }^{\S}$

2 National Institute for High Energy Physics, NIKHEF, and University of Amsterdam, NL-1009 DB Amsterdam, The Netherlands

3 University of Michigan, Ann Arbor, MI 48109, USA

4 Laboratoire d'Annecy-le-Vieux de Physique des Particules, LAPP,IN2P3-CNRS, BP 110, F-74941 Annecy-le-Vieux CEDEX, France

5 Johns Hopkins University, Baltimore, MD 21218, USA

6 Institute of Physics, University of Basel, CH-4056 Basel, Switzerland

7 Institute of High Energy Physics, IHEP, 100039 Beijing, China ${ }^{\triangle}$

8 Humboldt University, D-10099 Berlin, FRG $^{\S}$

9 University of Bologna and INFN-Sezione di Bologna, I-40126 Bologna, Italy

10 Tata Institute of Fundamental Research, Bombay 400 005, India

11 Boston University, Boston, MA 02215, USA

12 Northeastern University, Boston, MA 02115, USA

13 Institute of Atomic Physics and University of Bucharest, R-76900 Bucharest, Romania

14 Central Research Institute for Physics of the Hungarian Academy of Sciences, H-1525 Budapest 114, Hungary ${ }^{\ddagger}$

15 Massachusetts Institute of Technology, Cambridge, MA 02139, USA

16 INFN Sezione di Firenze and University of Florence, I-50125 Florence, Italy

17 European Laboratory for Particle Physics, CERN, CH-1211 Geneva 23, Switzerland

18 World Laboratory, FBLJA Project, CH-1211 Geneva 23, Switzerland

19 University of Geneva, CH-1211 Geneva 4, Switzerland

20 Chinese University of Science and Technology, USTC, Hefei, Anhui 230 029, China $\triangle$

21 SEFT, Research Institute for High Energy Physics, P.O. Box 9, SF-00014 Helsinki, Finland

22 University of Lausanne, CH-1015 Lausanne, Switzerland

23 INFN-Sezione di Lecce and Universitá Degli Studi di Lecce, I-73100 Lecce, Italy

24 Los Alamos National Laboratory, Los Alamos, NM 87544, USA

25 Institut de Physique Nucléaire de Lyon, IN2P3-CNRS,Université Claude Bernard, F-69622 Villeurbanne, France

26 Centro de Investigaciones Energeticas, Medioambientales y Tecnologicas, CIEMAT, E-28040 Madrid, Spainb

27 INFN-Sezione di Milano, I-20133 Milan, Italy

28 Institute of Theoretical and Experimental Physics, ITEP, Moscow, Russia

29 INFN-Sezione di Napoli and University of Naples, I-80125 Naples, Italy

30 Department of Natural Sciences, University of Cyprus, Nicosia, Cyprus

31 University of Nijmegen and NIKHEF, NL-6525 ED Nijmegen, The Netherlands

32 California Institute of Technology, Pasadena, CA 91125, USA

33 INFN-Sezione di Perugia and Universitá Degli Studi di Perugia, I-06100 Perugia, Italy

34 Carnegie Mellon University, Pittsburgh, PA 15213, USA

35 Princeton University, Princeton, NJ 08544, USA

36 INFN-Sezione di Roma and University of Rome, "La Sapienza", I-00185 Rome, Italy

37 Nuclear Physics Institute, St. Petersburg, Russia

38 University and INFN, Salerno, I-84100 Salerno, Italy

39 University of California, San Diego, CA 92093, USA

40 Dept. de Fisica de Particulas Elementales, Univ. de Santiago, E-15706 Santiago de Compostela, Spain

41 Bulgarian Academy of Sciences, Central Lab. of Mechatronics and Instrumentation, BU-1113 Sofia, Bulgaria

42 Center for High Energy Physics, Adv. Inst. of Sciences and Technology, 305-701 Taejon, Republic of Korea

43 University of Alabama, Tuscaloosa, AL 35486, USA

44 Utrecht University and NIKHEF, NL-3584 CB Utrecht, The Netherlands

45 Purdue University, West Lafayette, IN 47907, USA

46 Paul Scherrer Institut, PSI, CH-5232 Villigen, Switzerland

47 DESY-Institut für Hochenergiephysik, D-15738 Zeuthen, FRG

48 Eidgenössische Technische Hochschule, ETH Zürich, CH-8093 Zürich, Switzerland

49 University of Hamburg, D-22761 Hamburg, FRG

50 National Central University, Chung-Li, Taiwan, China

51 Department of Physics, National Tsing Hua University, Taiwan, China

$\S$ Supported by the German Bundesministerium für Bildung, Wissenschaft, Forschung und Technologie

$\mp$ Supported by the Hungarian OTKA fund under contract numbers T019181, F023259 and T024011.

ๆ Also supported by the Hungarian OTKA fund under contract numbers T22238 and T026178.

b Supported also by the Comisión Interministerial de Ciencia y Technología.

\# Also supported by CONICET and Universidad Nacional de La Plata, CC 67, 1900 La Plata, Argentina.

Ł Supported by Deutscher Akademischer Austauschdienst.

$\diamond$ Also supported by Panjab University, Chandigarh-160014, India.

$\triangle$ Supported by the National Natural Science Foundation of China. 
(a)

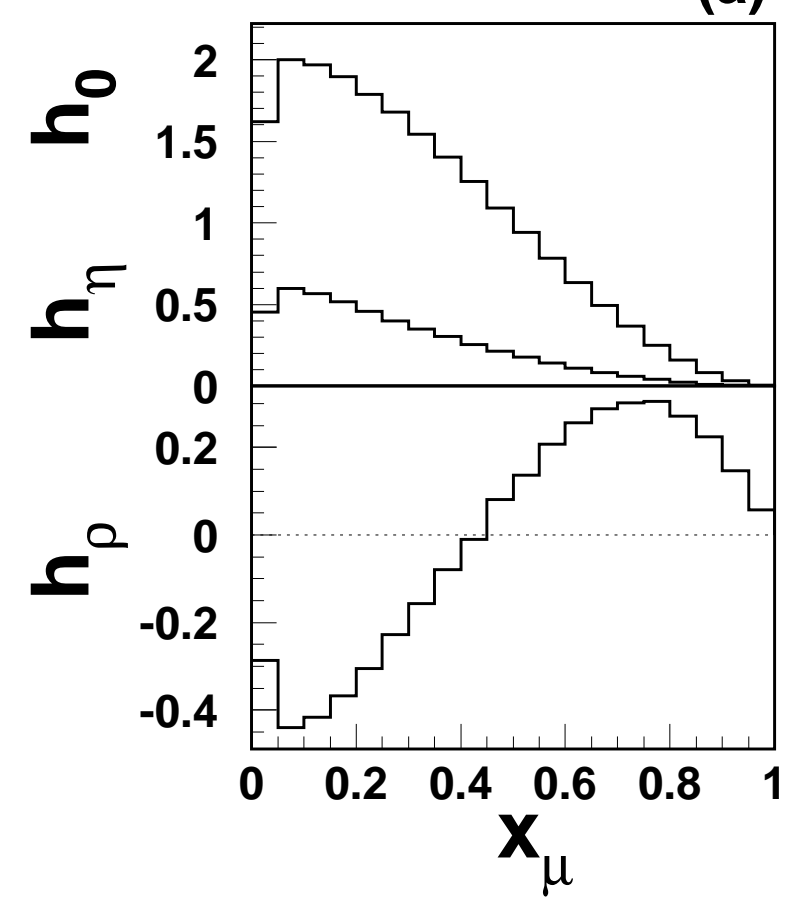

(b)

(c)
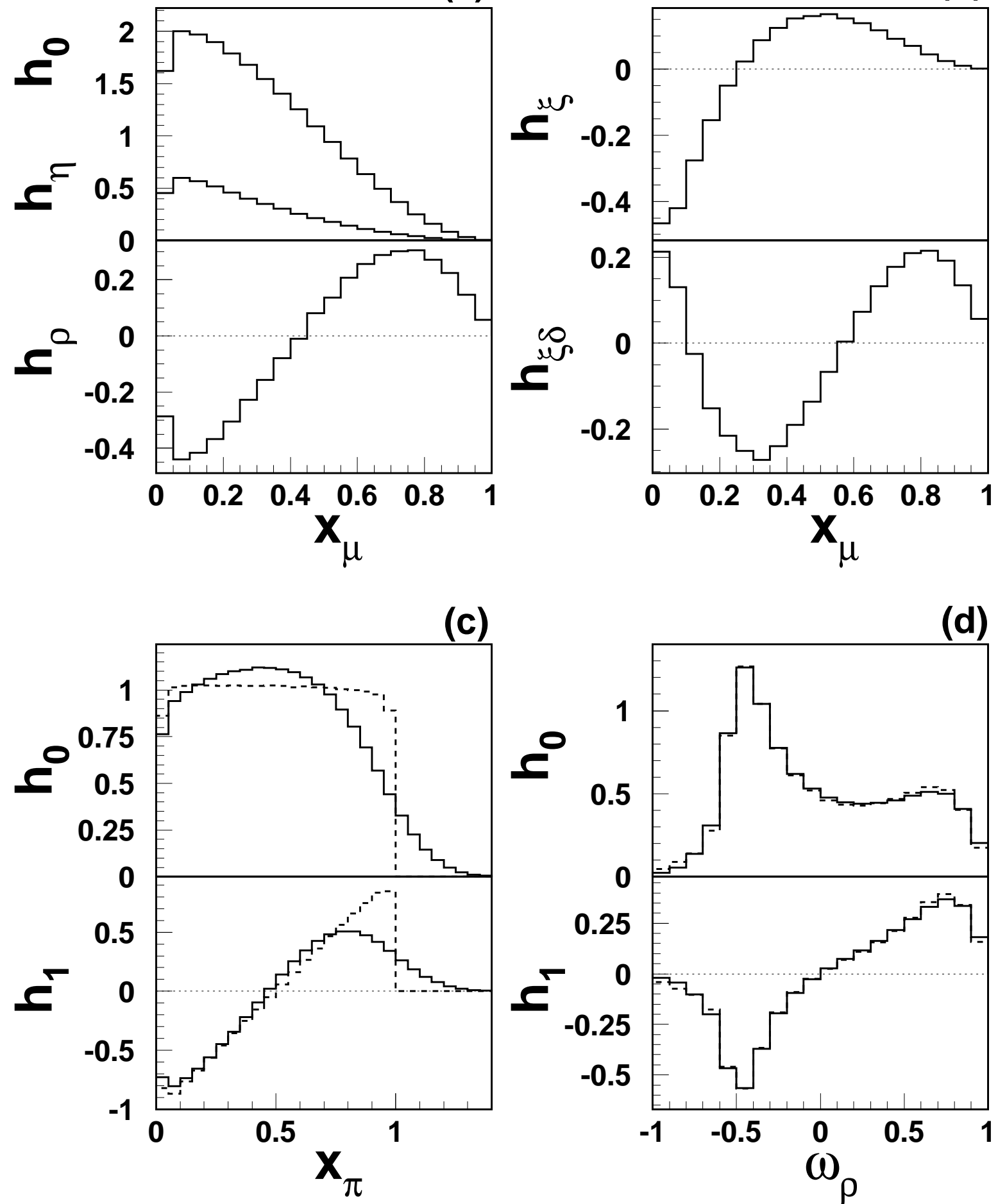

(d)

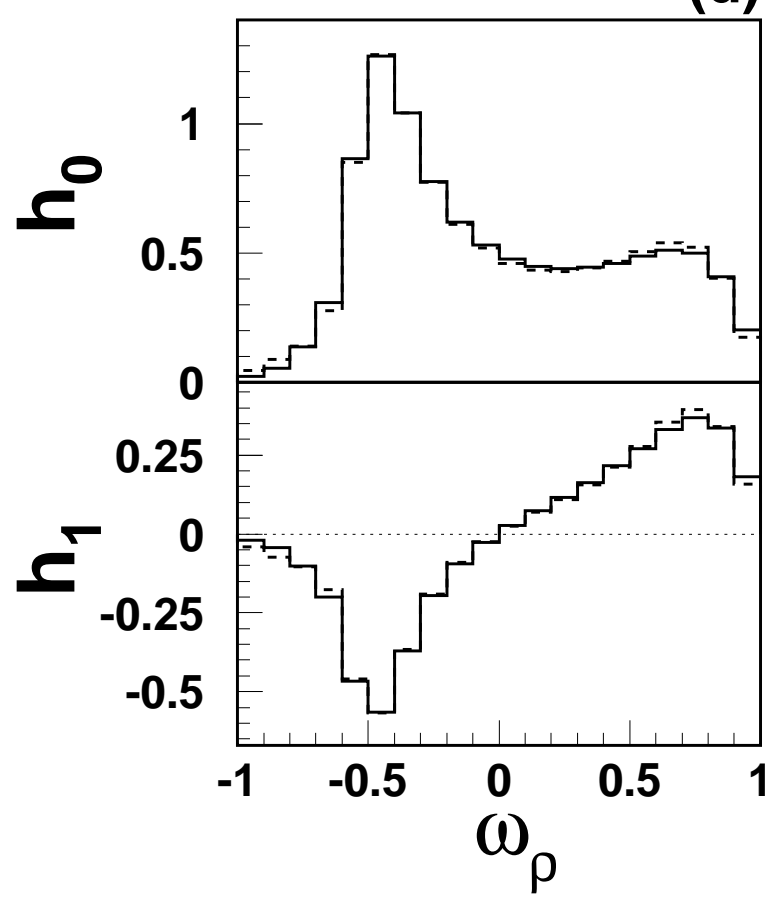

Figure 1: Kinematical functions used to fit (a)(b): $\mu$ spectra from $\tau^{-} \rightarrow \mu^{-} \nu_{\tau} \bar{\nu}_{\mu},(\mathrm{c}): \pi$ spectra from $\tau^{-} \rightarrow \pi^{-} \nu_{\tau}$ and $(\mathrm{d}): \rho$ spectra from $\tau^{-} \rightarrow \rho^{-} \nu_{\tau}$. The functions used to fit electron spectra from $\tau^{-} \rightarrow \mathrm{e}^{-} \nu_{\tau} \bar{\nu}_{e}$ are very similar to (a) and (b) apart from $h_{\eta}^{\mathrm{e}}$ which is suppressed by a factor of $\left(m_{\mathrm{e}} / m_{\mu}\right)^{2}$ relative to $h_{\eta}^{\mu}$. In (c) and (d), the dashed histograms correspond to generator level Monte Carlo; the solid histograms include detector resolution and acceptance. For (a) and (b), the effect of resolution and acceptance on the curves is not visible on this scale. 

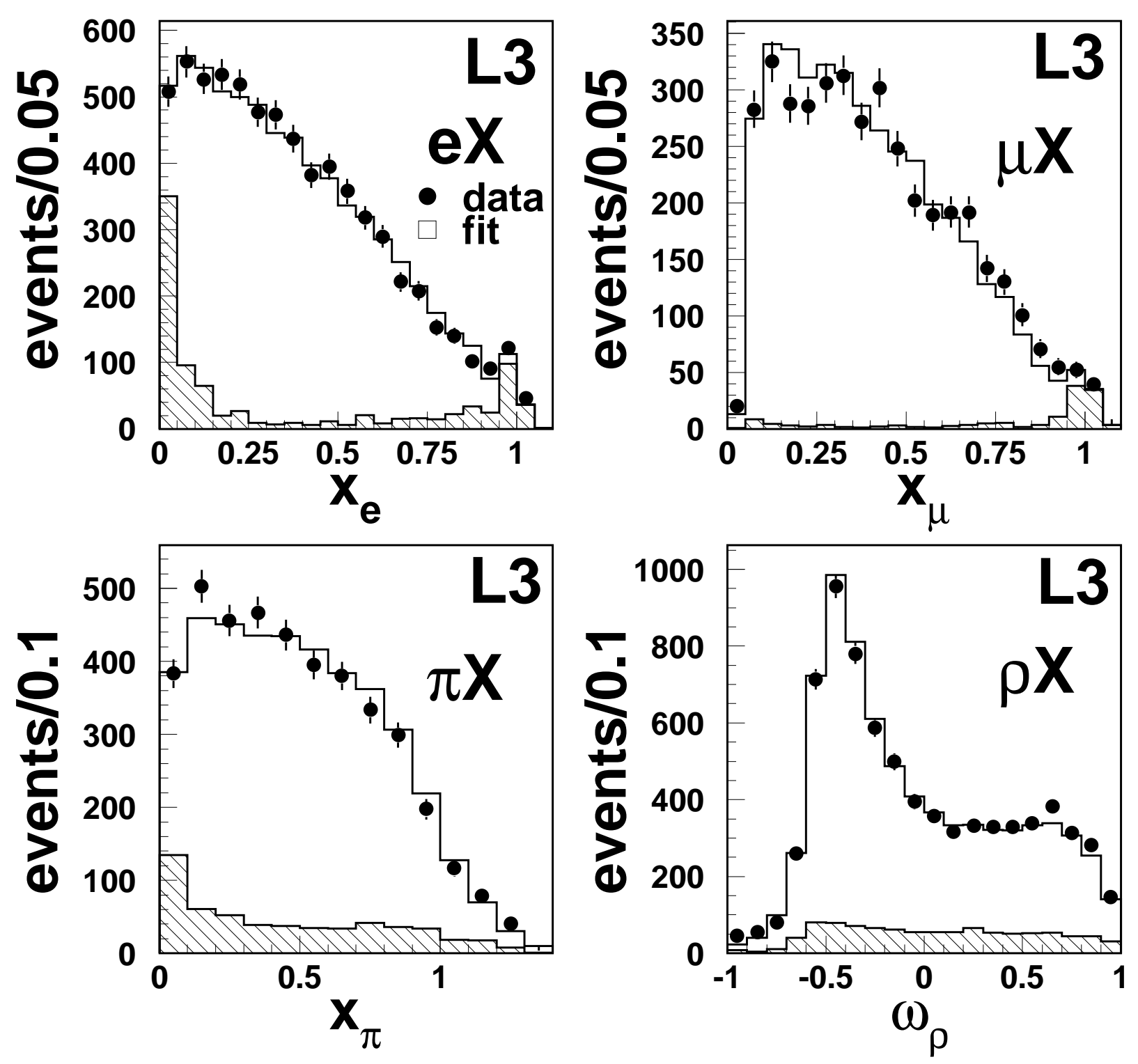

Figure 2: 1994-95 data and fit result for the $e X, \mu X, \pi X$ and $\rho X$ final states, where $X$ represents an unidentified $\tau$ decay. The shaded histograms show the sum of $\tau$ and non- $\tau$ background. 

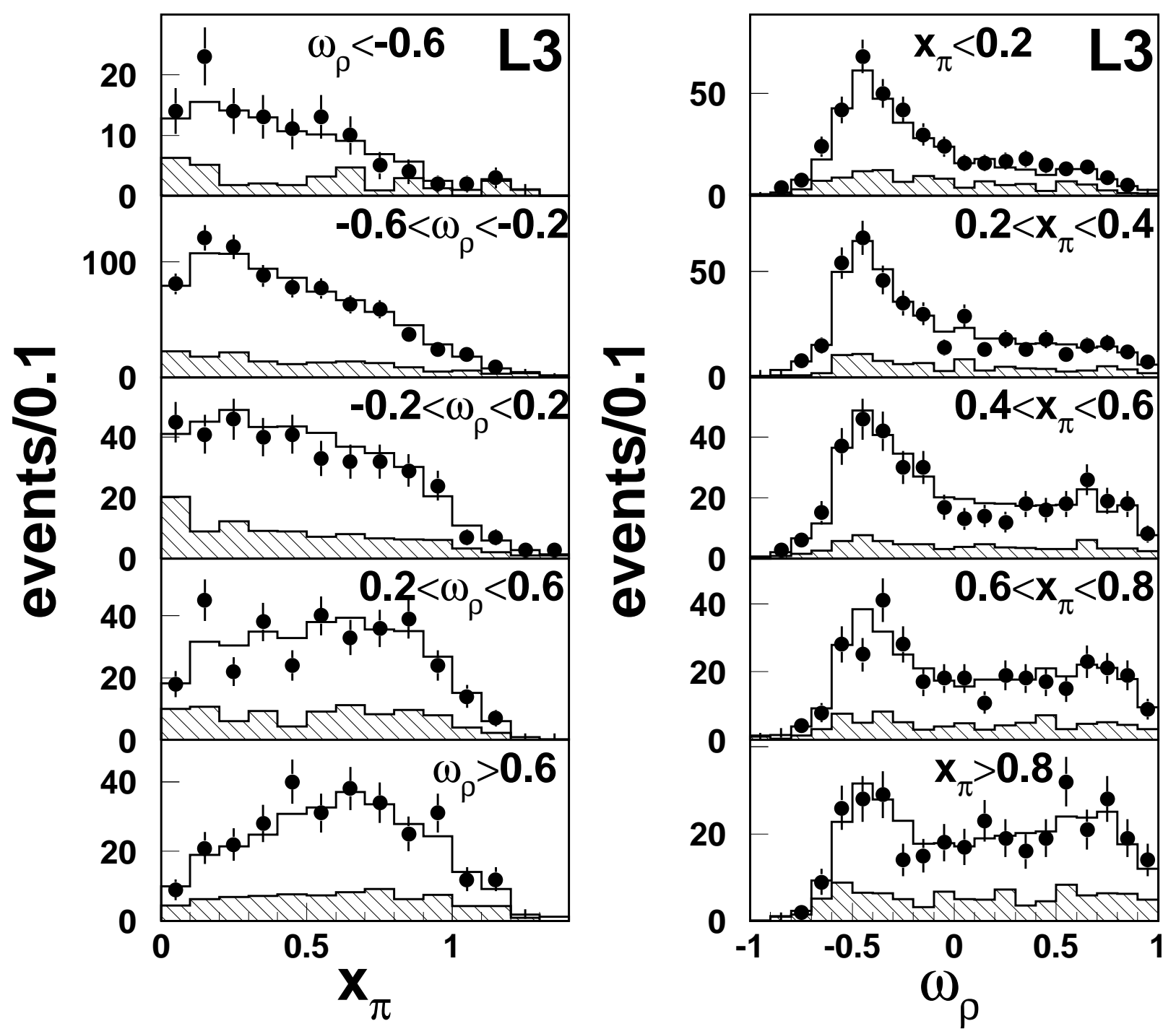

Figure 3: 1994-95 data (points) and fit result (histogram) in the $\pi \rho$ channel. On the left are pion energy spectra for different slices of the $\omega_{\rho}$ variable. On the right are distributions of $\omega_{\rho}$ for different slices in pion energy. The shaded histograms show the sum of $\tau$ and non- $\tau$ background. 